\title{
Unbeständigkeitsstrategien in Rechtssystemen der Peripherie: Eine Form alternativen Rechts ${ }^{1}$
}

\author{
Von João Maurício L. Adeodato
}

Drei Vorbemerkungen sollen am Anfang stehen, um die hier verfolgte Perspektive zu verdeutlichen.

Erstens setzen die folgenden Überlegungen einen sehr breiten, extensiven Begriff des Rechts voraus: Da, wo eine soziale Ordnung durch Normen gehalten wird, Normen, deren Nichtbeachtung erzwingbare Sanktionen verursachen können, gibt es Recht. Ordnung, Normen und die Möglichkeit von organisiertem Zwang werden hier als ausreichend angenommen, um Recht zu erzeugen. In gleicher Weise werden Begriffe wie Justiz oder die Vorstellung vom moralischen Inhalt juristischer Normen als undefinierbar ausgeschlossen. Vorgezogen wird demgegenüber der Begriff der Wirksamkeit - der Normen und der Rechtsordnung. Gerechtigkeit, Geltung und Legitimität gehen hier als eine Art persönliche Bewertung über das wirkliche, faktische Recht ein - nur wenn man mit dem Aufbau der Normen und Sanktionen einverstanden ist, wird das System "gerecht", "legitim" und derart genannt ${ }^{2}$.

Zweitens ist es weder Absicht noch Hoffnung dieser Ausführungen, das existierende Rechtssystem Brasiliens zu optimieren, sondern lediglich, eine tatsächliche Situation zu schildern; dies gleichwohl, da unvermeidlich, unter einer Theorie oder Philosophie des Rechts. Anders gesagt soll unsere Perspektive nicht prospektiv oder präskriptiv, sondern, auf eine viel bescheidenere und anspruchslosere Weise, lediglich retrospektiv und deskriptiv sein.

Drittens wird angenommen, daß Gesellschaften, die die Peripherie des gegenwärtigen Kapitalismus bilden und eher unpräzise "unterentwickelt" genannt werden, eine spezifische Form des Rechtes aufweisen. Ziel soll es sein, alternative Formen positiven Rechts herauszuarbeiten, welche in einer Art freundlichem Pluralismus mit jenem koexistieren, was man staatlich-dogmatisches Recht nennen kann. Innerhalb dieser Ausprägungen alternativen Rechts wollen wir jene Verfahrensweisen verfolgen, die vom Staat und den Regierungs-

Herrn Johannes Liebrecht gilt mein besonderer Dank für seine weit mehr als sprachliche Hilfe.

Dies heißt natürlich nicht, daß derlei Bewertungen keiner Verallgemeinerung zugänglich wären, um als Maßstab der Beurteilung von juristischen Ordnungen zu dienen. 
organen selbst praktiziert werden, obschon sie sich deutlich neben das staatlich-dogmatische Recht stellen, manche sogar contra legem. Diese hochgradig inkohärente Vermischung beinhaltet sicherlich Probleme, aber sie funktioniert, wie man feststellen kann.

Dies tut sie freilich auf eigene Weise. Vielfach wurde über eine Krise des Rechts und eine Krise des Staates in gegenwärtigen peripheren Gesellschaften gesprochen; doch vielleicht sollte ein permanenter Krisenzustand nicht wirklich als Krise, vielmehr als eine Organisationsform verstanden werden, welche für manchen Beobachter chaotisch zu sein scheint, die aber dennoch sehr effizient operiert. Für unsere Zwecke mag Brasilien diese Situation gut veruzutlichen. Hier arbeiten die sozialen Subsysteme je gegeneinander und kommen sich nicht selten in die Quere. So spielt, als Beispiel, die wirtschaftliche Lage der Gesellschaft und auch von Individuen eine zentrale Rolle sowohl in der Rechtsanwendung als auch für die Legitimierung von Regierungen; ebenfalls können politische Kasuistik und Freundschaftsbeziehungen den Gang staatlicher Entscheidungen modifizieren, ein Umstand, der durch ein altes Sprichwort illustriert wird: "Den Freunden alles, den Gegnern das Gesetz". Recht wird politisiert oder, mehr noch, allopoietisch ${ }^{3}$. An der Stelle einer Kultur von Rollendifferenzierung und bürokratischer Unparteilichkeit steht eine Kultur des Begünstigens und der Politik "guter Nachbarschaft", an der Stelle der staatlichen Effizienz die Verehrung von Familie, Privilegien und Korporatismus. Gleichwohl erscheint es noch immer als ein traditioneller Gemeinplatz zu denken, daß Modelle, die für das Verstehen und den Umgang sehr verschiedener empirischer Situationen entworfen wurden, generalisiert werden dürften ${ }^{4}$. Vermutlich spielt unter den vielen Schwierigkeiten, den stets konstatierten Krisenzustand zu verstehen und zu beschreiben, gerade die Anmaßung, auf alle Länder das Modell eines eurozentrisch-modernen Staates auszuweiten, eine entscheidende Rolle. Denn viele Juristen und andere Sozialwissenschaftler scheinen von einer Art ontologischer Epistemologie auszugehen, dem Glauben, daß es möglich sei, eine universale Theorie zu entwerfen, die ein klares Leitbild für Recht, Politik, die sozialen Verhältnisse oder ihre Entwicklungs- und Evolutionsformen entdecken könnte. Es weisen aber diese Kriterien gewöhnlich eine starke Bindung an das auf, was man als die historische Erfahrung im Wohlfahrtsstaat beobachten kann; und ein solcher liegt leider nicht immer vor.

Für den Begriff der autopoiesis (und allopoiesis) vgl. Humberto R. Maturana / Francisco J. Varela, Autopoiesis and Cognition - The Realization of the Living, Dordrecht 1980; Niklas Luhmann, Die Wirtschaft der Gesellschaft, Frankfurt a.M. 1988, S. 43 ff. (Kap.II); zu seinem Einfluß in der Rechtstheorie s. Gunther Teubner (ed.), Autopoietic Law: A New Approach to Law and Society, Berlin / New York 1988; ders., Recht als autopoietisches System, Frankfurt a.M. 1989; Karl-Heinz Ladeur, "Abwägung" - Ein neues Paradigma des Verwaltungsrechts, Frankfurt a.M. I New York 1984.

Das ist auf verschiedenen Gebieten zu beobachten, so der Wirtschaft, der Stadtplanung, dem Ingenieurwesen, der Medizin und auch dem Recht. 
Ein Kontrast zu den Hauptströmungen der zeitgenössischen Rechtsphilosophie ist also Anliegen dieses Beitrags, denn eine Theorie muß sich den konkreten Gegebenheiten ihres jeweiligen Gegenstandes anpassen: In peripheren Gesellschaften erlangen grundlegende Axiome der von ihnen importierten Rechtssysteme nur schwankende, oft kaum voraussehbare Wirksamkeit - so die Prinzipien der Normenhierarchie und des Vorrangs der Verfassung, das Grundrecht auf Gleichheit vor dem Gesetz oder die Einhaltung der gerichtlichen Neutralität. Wählen wir daher einen eher problemorientierten denn systemischen Zugang, so werden wir die Unausgeglichenheit zwischen dem an der Gesetzgebung bzw. am gerichtlichen Prozeß orientierten Modell eines durch den Staat zur Geltung gelangten (und angenommenerweise allzuständigen) dogmatischen Rechts auf der einen Seite und den Verfahrensweisen für die Behandlung rechtlicher Konflikte, wie sie tatsächlich und wirkungsvoll stattfinden, auf der anderen Seite besser wahrnehmen können. Möglicherweise ist der dogmatische Weg, rechtliche Konflikte zu entscheiden, unter pragmatischen, demokratischen, ethischen oder auch wissenschaftlichen Gesichtspunkten wünschenswerter und angemessener. Ungeachtet der Wichtigkeit dieser Fragen sollen sie hier aber nicht unser Gegenstand sein. Sondern es wird jenem für periphere Länder so entscheidenden Spielraum nachgegangen, in dem sich abseits der offiziellen Ordnung sehr unbeständige, marginale Verfahren konstituieren, die eigene Strategien für die Kontrolle einer scheinbar chaotischen Organisation anbieten.

\section{Der hier verwendete Begriff von "dogmatischem Recht"}

Rechtsdogmatik ist ein Begriff, der die überwiegende Form des Rechts in modernen Staaten bezeichnet. Offensichtlich hat diese Form der Verteilung staatlicher Gerechtigkeit nicht immer existiert, sondern umfaßt ein historisches Phänomen ohne Beispiel. Wenn auch dieser Prozeß der Dogmatisierung positiven Rechts innerhalb der Mehrheit gegenwärtiger Gesellschaften noch nicht vollendet ist, so erscheint doch der Versuch, ihn voranzutreiben, als eine generelle Tendenz. Dieses dogmatische Recht erfordert, neben anderen Charakteristika, das Vorhandensein einer komplexen Gesellschaft mit einem stabilen bürokratischen Apparat. Zwei seiner Haupterfordernisse seien vorab hervorgehoben.

Zunächst der Zwang, auf der Grundlage vorgegebener Normen zu argumentieren, angeblicherweise eine Komponente der Rechtsordnung. Jeder, der dogmatisch argumentiert, muß seine Argumente auf eine Rechtsnorm gründen, die als solche durch das System selbst definiert oder angezeigt wurde. Ein Anwalt von Slumbewohnern, die anderer Leute Eigentum besetzt halten, kann nicht argumentieren, daß der Eigentümer dieses oder jenes Eigentumsrecht nicht habe, nur weil er reich sei und ja schon so viel Land habe, - obwohl das Argument rational, intelligibel und sogar sehr plausibel ist. Es gibt keine solche Norm in der rechtsstaatlichen Ordnung Brasiliens. Sofern er die staatliche Zwangsgewalt auf seiner Seite wissen will, darf der Anwalt (oder auch ein Richter, der auf Konflikte um die Agrar- 
reform eingehen möchte) seine rhetorischen Rechtfertigungen nur auf Normen gründen, die etwa auf die Sozialbindung des Eigentums oder einen Mißbrauch dessen verweisen, sind doch solche wenigstens in der Verfassung ausdrücklich formuliert. Das ist vermutlich das wichtigste Dogma dieses Rechts: Jeder, der die Zwangsgewalt des Staates braucht oder anstrebt, muß die Sprache des Staates sprechen.

Der Zwang zu entscheiden, das Entscheidungsverweigerungsverbot, ist das zweite dogmatische Prinzip, das hier hervorzuheben ist, - das Verbot dessen, was die Römer non liquet nannten. Nicht nur behauptet der moderne Staat ein Rechtsmonopol; dogmatisches Recht soll auch jeglichen Rechtskonflikt entscheiden ${ }^{5}$. Ein Richter muß ein Urteil fällen, selbst wenn er meint, daß nicht genügend Anhaltspunkte für eine gerechte Entscheidung vorliegen. Das war nicht immer so. Salomo handelte bekanntlich weise, als er drohte, das umkämpfte Kind in zwei Teile schneiden zu lassen, da der Mangel an Beweisen es ihm nicht erlaubte, sich eine Meinung zu bilden ${ }^{6}$. Man stelle sich einen modernen Richter vor, der anordnet, das strittige Objekt zu zerstören, weil es keine genauen Beweise oder Normtexte gebe, die den Eigentumsanspruch einer der Parteien auf die Sache rechtfertigten ... doch zu Salomos Zeit war Recht noch nicht dogmatisch organisiert.

Es ist freilich kein neuer Gedanke, daß das staatliche Rechtsmonopol asymmetrisch und weit eher dogmatisch fingiert als faktisch wirkungsvoll ist. Diese These wurde bereits von vielen soziologischen und rechtlichen Schulen des 19. Jahrhunderts vertreten, wie der Historischen Schule und der Freirechtsbewegung in Deutschland oder der École de la Libre Recherche Scientifique in Frankreich. In einem natürlich anderen Kontext haben auch periphere Staaten der Gegenwart ein dogmatisches Recht nicht aufbauen können, entgegen aller dahingehender Behauptungen und Legalfiktionen. Demgemäß entstehen Verfahren der Entscheidungsfindung, die nicht zu der herkömmlich-generellen Theorie rechtlicher Dogmatik und Soziologie passen und durch sie auch nicht erklärt werden können ${ }^{7}$. Sind sie Recht? - Ubi societas ibi ius, nach diesem Sprichwort wohnt einem menschlichen Zusammenleben stets der Kontext, den wir Recht nennen, schon inne, gibt es keine Gemeinschaft (selbst die primitivste nicht) ohne Recht (- was kaum der Eschatologie eines orthodoxen Marxismus den Mund redet). Betrachten wir dagegen den staatlichen Anspruch auf das Monopol für die Schaffung von Rechtsnormen, so müßte eine große Anzahl von Brasilia-

Eine zweif elhafte Perspektive auf dieses Problem hat eine Variante des juristischen Positivismus zur Identifikation von Recht und Staat geführt: Hans Kelsen, Reine Rechtslehre, 2. Aufl. Wien 1960 (Nachdr. 1983), Nr. 41 (S. 289 - 320).

6 1. Kön. 3, $16-28$.

Dies habe ich bereits dargelegt: A Legitimação pelo Procedimento Juridicamente Organizado Notas à Teoria de Niklas Luhmann, in: Revista da Faculdade de Direito de Caruaru 16 (1985), S. 85 f. Vgl. auch Niklas Luhmann, Das Recht der Gesellschaft, Frankfurt a.M. 1995, S. 25, 81, 194 und 584 . 
nern und viele andere Menschen ohne Recht leben. Offensichtlich jedoch werden ihre unvermeidlichen Konflikte durch ein anderes (in diesem Sinne, alternatives') Recht außerhalb des Staates aufgefangen und neutralisiert. Dies ist eine direkte Folge aus dem Umstand, daß es peripheren Staaten nicht gelungen ist, ihre Gerechtigkeit an alle Bürger auszuteilen. Wenn nicht sie entscheiden - und Konflikte müssen entschieden werden -, werden notwendigerweise andere Instanzen diese soziale Funktion erfüllen.

\section{Dogmatisches Recht und periphere Länder}

Die Vielfalt der Rechtsquellen ist über Jahrzehnte erforscht worden, als Teil der Diskussion um Pluralismus im Recht und das beanspruchte Staatsmonopol für die Schaffung positiven Rechts. In Gerichtswesen unterentwickelter Gesellschaften, die unter anderem von einem ineffektiven Staat geprägt sind, ist dieser Fragenkreis entscheidend und wirft ein Licht auf die Austeilung von Gerechtigkeit wie auch die Legitimation der Regierungen ${ }^{8}$ :

Kongruenz oder Konsistenz, als Übereinstimmung mit inneren Normen, scheinen offenbar keine wesentlichen Bestandteile des Systembegriffs zu bilden, insbesondere was soziale Systeme betrifft. Dennoch pflegen normative Inkompatibilitäten auf lange Dauer zu nachteiligen Folgen zu führen. Und aus diesem Grunde entwickelte die Rechtsdogmatik ein hermeneutisches Instrumentarium, um systemischen Inkongruenzen zu begegnen. Derlei Strategien wurden zwar auch formell in die rechtlichen Ordnungen peripherer Länder übernommen. In diesen Gesellschaften entfalten jedoch, wie erwähnt, Grundannahmen eurozentrischer Rechtssysteme keine Effizienz.

Wird demgemäß erkannt, daß Allopoiesis sehr wohl in sozialen Organisationen durchführbar und Kongruenz in keiner Weise für den Bestand von Rechtssystemen essentiell ist (welche weit mehr Antinomien mit sich führen, als Juristen gewöhnlicherweise sich zuzugeben bereit sind), so gelangt man zu einer Kritik der z.T. unvorsichtigen Universalisierungen von sog. "rationalistischen" Theorien und anderen allumfassenden Theoriesystemen, welche nicht passend wirken, um jene Interaktionen zu verstehen, die wir befragen möchten. Die Strukturen der in alternativem Recht geführten Argumentationen sind - entgegen den meisten dieser Theorien - ausgesprochen nebensächlich und zufällig. Theorieansätze, die auf den Begriffen einer praktischen Vernunft beruhen, sehen die eigene Universalität

Siehe João M. Adeodato, Brasilien. Vorstudien zu einer emanzipatorischen Legitimationstheorie für unterentwickelte Länder, in: Rechtstheorie 22 (1991), S. 108 - 128; sowie ders., Practical Regularities in Underveloped Countries, in: Robert Alexy / Ralf Dreier (Hrsg.), Rechtsystem und praktische Vernunft / Legal System and Practical Reason, Stuttgart 1991 (ARSP Beiheft 51), S. $62-67$. 
offensichtlich als selbstverständlich an, - nicht als ein Forschungsresultat, sondern als ein vorausgenommenes Postulat?

Dies scheint an gegenwärtigen peripheren Gesellschaften belegt werden zu können. Die Toleranzgrenzen zu intrasystemischen Widersprüchen, wenigstens innerhalb des rechtlichen Subsystems, zeigen sich weit flexibler als der orthodoxe Marxismus, Abhängigkeitstheorien und andere generalisierende Theorien bisher erwarteten. Es ist wohl auch ein Vorurteil zu denken, daß diese außerdogmatischen Wege der Konfliktlösung Dysfunktionen des Rechtssystems seien. In der Tat werden sie begünstigt durch Dysfunktionen im staatlichen Rechtssystem, das das Monopol für die Erzeugung von Rechtsnormen beansprucht, doch helfen sie durchaus auch, das Rechtssystem aufrechtzuerhalten, indem sie Konflikte kontrollieren. Diesen Inkonsistenzen wird in ausschließlich dogmatischen Argumentationen gewissermaßen ausgewichen, wohl da die offene Annahme ihrer Existenz eine nicht legitimierbare Wirkung zeitigen könnte. Allerdings sind diese stillschweigenden Regeln sehr wohl deutlich für alle Beteiligten.

Da solche Strategien nicht zu einem autopoietischen und durch den Staat symmetrisch positivierten Subsystem gehören, verbinden sie rechtliche und außerrechtliche Aspekte, was natürlich einen präzisen Zugang zu ihnen ausgesprochen erschwert. Demgemäß bedeutet die Verflochtenheit des Rechts mit der es umgebenden Welt aber auch, daß staatliches Recht nicht bloß der nichtsstaatlichen Entscheidungsinstanzen, sondern sogar anderer nichtrechtlicher Subsysteme wie der Religion, Wirtschaft, Freundschaft, Moral etc. bedarf. Derartige verschränkt-normative Beziehungsfelder nehmen dem ineffizienten Staat eine Aufgabe ab, für deren Handhabung er augenscheinlich über keinerlei Fähigkeit verfügt, nämlich: als die einzig legitime Rechtsquelle die Lösung rechtlicher Konflikte zu monopolisieren.

Zunächst sei eine angemessene Theorie dieses peripheren Rechts durch eine beschreibende Soziologie darüber vorbereitet, wie Rechtskonflikte in diesen Gesellschaften wirkungsvoll entschieden werden. Ihr Gegenstand dürfte kaum auf die Rechtsfiguren unter staatlichem Vormund reduziert werden (Gesetze, praeter legem mores, Gerichtsentscheidungen, rechtmäßige Verträge und andere Formen staatlich akzeptierter Rechtsnormen), sondern muß gerade jene marginalen Strategien einschließen, die vom staatlichen Recht ignoriert oder

So Michel Paroussis, Die transkulturelle Ubiquität des Reasonable Man. Zur Universalität des Vernünftigen als Postulat rechtsethnologischer Forschung, in: Rechtstheorie 23 (1992), S. 123 140; vgl. auch bei Niklas Luhmann, Soziale Systeme. Grundriß einer allgemeinen Theorie, Frankfurt a.M. 1984, S. 9. Diese allumfassenden Theorien und Vorschläge helfen dem Richter nicht, seine Entscheidung zu treffen; sie können allerdings dazu dienen, die durch lange, teils schiefe und irrationale Umwege getroffene Entscheidung darzustellen und zu begründen; s. hierzu Katharina Sobota, Don't Mention the Norm!, in: International Journal for the Semiotics of Law 4 (1991), S. $45-60$. 
sogar verboten werden. Es wird sich so erweisen, daß alternatives Recht nicht nur Rechtslücken in praeter legem-Form auffüllen kann (etwas, das selbst die radikalste dogmatische Theorie akzeptieren wird), sondern sogar oft dem gesetzten Recht widerspricht, also contra legem vorgeht. Dies soll näher betrachtet werden.

\section{Peripheres Richterrecht und Verfassungsauslegung als eine Form alternativen Rechts}

Ohne Zweifel finden sich eine ganze Reihe rechtlicher Normen, die in keiner Weise Staat oder Regierung betreffen, sondern nur in einzelnen Gegenden gelten, in welchen der Staat, wenn überhaupt, bloß in strafrechtlichen Belangen auftritt. Der übrigen Rechtsfälle (wie Nachbarschaftskonflikte, solcher um Eigentumsgrenzen oder Streitfälle im familien- und vertragsrechtlichen Bereich) nehmen sich die Führer von Anwohnervereinen, Pfarrer oder auch - vom Staatsgesichtspunkt - kriminelle Organisationen an. Wir könnten es alternatives Recht stricto sensu nennen; dieses Recht, ein Produkt nichtdogmatischer Quellen, ist im besonderen Hinblick auf die brasilianischen favelas vielfach untersucht worden ${ }^{10}$. Die Außenseiter bilden und leben ihr eigenes Recht, oftmals im Kampf gegen die staatliche Ordnung. Dabei scheint dieses marginale Recht kaum ein politisches Ziel zu verfolgen. Trotzdem sind einige dieser Randgruppen in großem Maße organisiert und können sogar eine tragende soziale Rolle übernehmen, insbesondere zwischen den ausgeschlossenen Massen und den korrupten Sektoren innerhalb der offiziell-legalen Gesellschaft Verbindungskanäle schaffen. Sie schöpfen auf diese Weise, den staatlichen Institutionen zum Trotz, ihre eigenen juristischen Verfahren.

Die Strategien, die wir hier näher beleuchten wollen, gehören jedoch einem mit dem rein staatlichen Handeln dicht verknüpften Rechtssystem an und befinden sich in enger Abhängigkeit zu den Regierungsinstitutionen, obgleich sie in fast gänzlicher Autonomie zum staatlich-dogmatischen Recht stehen. Diese Verfahren der Entscheidungsfindung werden von staatlichen Organen selbst geführt, können aber sehr wohl grundlegenden Verfassungsprinzipien entgegenlaufen. Alternatives Recht findet sich so nicht nur jenseits des Staates, sondern ebenso innerhalb seiner. Unsere Forschungsgruppe in Brasilien richtet ihr Hauptaugenmerk auf die von der Judikative des Bundesstaates Pernambuco gefällten Entscheidungen, muß also eben diesen außerdogmatischen Verfahren innerhalb des staatlichen Handelns Rechnung tragen.

Drei Aspekte dieser Untersuchungen lassen sich hier nennen: Zunächst ist da die (im hier gemeinten Sinne) dogmatische Perspektive auf die gesetzlichen Verfahren der Entschei-

10

Etwa bei Boaventura de Sousa Santos, The Law of the Opressed: The Construction and Reproduction of Legality in Pasargada, in: Law and Society Review 12 (1977), S. 5 - 126. 
dungsfindung gemäß dem "Wortlaut" oder "Geist" der materiellen und prozessualen Normen. Von ihr aus wird zuvor ermittelt, wie die staatliche Verteilung von Gerechtigkeit verwirklicht werden müßte, wenn das Verfahrensprinzip des ,Handelns vom Amts wegen in voller Weise effektiv wäre. Denn natürlich sind die Gerichtsverfahren bereits von den verschiedenen Verfahrensgesetzen und ihren Normen geregelt, deren Ketten von Anordnungen, Kostenübernahme, Vorladung, Einspruch etc. eigentlich durch den Staat garantiert werden sollen. Nun beobachten wir eine Hälfte der Prozesse aus unserem Untersuchungsfeld nur von außen, ohne auf sie einzuwirken, und legen die Reihenfolge ihrer dogmatischen Verfahrensabläufe ganz in die Hände amtlicher Initiative, so daß sich verfolgen läßt, wie die gerichtliche Bürokratie sich selbst überlassen vorgeht. Die andere Hälfte der Versuchsprozesse wird beeinflußt, indem von den Parteien die Absprache mit den zuständigen Justizbeamten, die persönliche Kontaktaufnahme zu ihnen gesucht und ihre Sympathie erworben wird - oder sie einfach bestochen werden. Sofern dies der Fall ist, versuchen wir nachzuzeichnen, welche Bereiche staatlichen Rechts übertreten wurden. Jedenfalls gelangen diese Prozesse zu einer weit schnelleren Entscheidung, die sogar Vorzüge aufweist.

Ein zweiter Schritt ist dann, die Gesetzgebung (oder auch ihr Nichtvorhandensein) zu erfassen, die das Entstehen marginaler Verfahrensweisen in der Rechtsprechung und deren Interpretation und Anwendung hervorruft; es handelt sich also um gesetzliches Recht, das, obgleich rechtsgültig und sozialwirksam, gegen Verfassungsnormen verstößt und Wirksamkeit wie Legitimität des Staates als Ganzes beeinträchtigt. Ein Beispiel hierfür wäre gewöhnliches Gesetzesrecht, das den Zugang zu öffentlichen Ämtern unter Umgehung des Leistungsprinzips einrichtet, obwohl in der Normenhierarchie höherrangiges Recht dies ausdrücklich verbietet, oder auch die zahlreichen Ausnahmen von den eigentlich einschlägigen Bestimmungen über öffentliche Auktionen; in gleicher Weise legalisierte Privilegien, Vetternwirtschaft, Korporatismus usw. Dieser Teil der Untersuchung richtet sich also auf die Erfassung solcher Gesetze und ihres Ursprungs innerhalb des Staates (ob im exekutiven, legislativen oder judikativen Bereich) sowie die Aufdeckung ihrer systematischen Widersprüche zu höherrangigem Recht, insbesondere zur Verfassung.

Schließlich untersuchen wir auf einer dritten Ebene Verfahren, die unzweideutig rechtswidrig sind, obgleich sie durch die gerichtliche Praxis selbst institutionalisiert werden und auch mit einer laisser-faire-Politik der Justizbürokratie rechnen können. Unverhoffterweise helfen diese Strategien manchmal, den Bestand des Systems zu sichern. Die gesetzlich verankerte Beweiserhebung (unter Berücksichtigung von Fristen, Formvorschriften, Urkunden etc.) mag sich als unangemessen herausstellen und leicht von effektiveren, wenn auch inkohärenten Strategien in den Schatten gestellt werden. Ein öffentlicher Beamter, ob Richter oder auch Rechtspfleger, wird den Beklagten nicht ohne ein vorheriges Bestechungsgeld vorladen, dessen Höhe gemäß dem Streitwert variieren kann; sich mit einem Notar oder Buchhalter zu überwerfen, bringt schnell Verzögerungen oder das Verschwinden von Beweisen und Urkunden etc. mit sich. Da, wie schon gesagt, der Grundsatz des 
Handelns von Amts wegen nur unregelmäßig greift, muß die rechtliche Bürokratie mit persönlichen Beziehungen, Freundschaften, vorgespielter Sorgfalt und der Einflußnahme (etwa auf das Tempo von Zwangsvollstreckungen) durch Draufgaben usw. in Gang gebracht, geschmiert werden.

Nicht zu vergessen sind zuletzt die Abwehrmaßnahmen, die in vereinzelten Fällen gegen die genannten Verfahrensstrategien getroffen werden, entweder durch den Staat selbst (durch die Corregedoria de Justiça, das zuständige Organ zur Ahndung von Korruption im Gerichtswesen), die Anwaltsvereinigung von Pernambuco (mittels einer eigens gegründeten Kommission) oder andere nichtstaatliche Organisationen. Ein etwaiger Erfolg solcher Bemühungen wird ebenfalls ausgewertet.

Soviel zu dem hier beispielhaft vorgestellten Programm unserer Forschungsgruppe in Recife. Das Interesse an alternativem Recht ist in Brasilien jedoch nicht auf Untersuchungen dieser Art beschränkt, sondern erstreckt sich über viele Äußerungen und Positionsnahmen auch in den Medien, die es aus je unterschiedlichem Hintergrund vertiefen wollen. So stand nicht nur die Bewegung selbst, das soziale Faktum, sondern auch der Sprachgebrauch des Ausdrucks "alternatives Recht" im Zentrum intensiver Debatten. Man könnte diese aktuelle Diskussion wie folgt zusammenfassen ${ }^{11}$ :

Eine Strömung ignoriert alternative Verfahren der Konfliktbewältigung einfach und betrachtet sie entweder als rechtlich irrelevante, lediglich soziale Praxis, als Gewohnheiten ohne Rechtskraft oder auch als fraglos rechtswidrig - sie versteht staatliches Recht als das einzige Recht. Ihr gemäß stellt eine Gerichtsentscheidung, welche einen des bewaffneten Bankraubes Angeklagten freispricht, weil er am Verhungern gewesen sei ${ }^{12}$, keinerlei Form des Rechts - ob alternatives oder wie auch immer geartetes - dar, sondern lediglich eine contra legem-Entscheidung, die aus der Rechtsordnung zu verbannen ist. Die Rechtsfigur des furto famélico ${ }^{13}$ auf einen rechtswidrigen Gewaltakt, etwa einen Raub, auszuweiten, sei ein klarer Mißbrauch am Recht. Diese Meinung wird freilich problematisch, wenn sogar bis hin zum höchsten Gerichtshof eindeutig gegen das Strafgesetzbuch entschieden wird; dies in einem Land, dessen Tradition nicht die des Common Law ist, und zumal im Strafrecht, für welches die Bindung an den Gesetzestext eine überragende Bedeutung einnimmt ${ }^{14}$.

João M. Adeodato, Para uma Conceituação do Direito Alternativo, in: Revista de Direito Alternativo 1 (1992), S. 157 - 174.

12 Solche Entscheidungen wurden nicht nur einmal gefällt; vgl. die Ausgabe der Zeitschrift Isto é/Senhor (São Paulo) vom 30.10.1991 (Nr. 1153), S. 7.

13

Der furto famélico bezeichnet den aus extremem Nahrungmangel motivierten und gewaltlos begangenen Diebstahl.

14

Obwohl der Wortlaut des braslianischen Strafgesetzbuches ausdrücklich vorsieht, daß der Beischlaf mit einem Mädchen unter vierzehn Jahren als Vergewaltigung zu bestrafen ist, entschied 
Eine weniger konservative Variante erkennt alternative Verfahren in ihrer prater legemWirkung an, so wenn ein Richter zu spontanen Formen des Alltagshandelns. greift, um Lücken oder Inkonsistenzen innerhalb des staatlichen Rechts auszugleichen. Das ist überdies in $\S 4$ des Einführungsgesetzes zum brasilianischen Zivilgesetzbuch vorgesehen, das 1942 verkündet worden und noch immer in Kraft ist. Einige Entscheidungen von Anwohnervereinen beispielsweise könnten vom Gerichtsapparat des Staates als legitime Rechtsformen bestätigt werden, die Gesetze der Rechtsgemeinschaft zu ergänzen. Die traditionelle Rechtslehre begegnet dieser Frage, indem sie mores als Rechtsquelle anerkennt, sofern sie nicht einen offensichtlich illegalen Charakter tragen.

Eine dritte Richtung betont mit dem Ausdruck "alternatives Recht" eine eigene ideologische Interpretationsweise und Anwendung staatlichen Rechts, die der , alternative Gebrauch von Recht' genannt wurde. Gemäß dieser Perspektive besteht die alternative Bewegung hauptsächlich in der kritischen Anwendung staatlicher Normen, insbesondere mit Hilfe von Lücken in der Gesetzgebung und hermeneutischen Techniken. Dieser Ansatz beinhaltet eine Zurückweisung vieler Grundannahmen des dogmatischen Positivismus, etwa der Neutralität von Rechtsnormen, des wissenschaftlichen Charakters ihrer Anwendung und der Unparteilichkeit des Richters. Diese Gruppe vertritt eine betonte Politisierung der staatlichen Gerichtsentscheidungen, doch will sie ihrem Anspruch nach die rechtlichen Grenzen des Staates nicht hinter sich lassen, vielmehr nach einer Optimierung statt Negation des Systems suchen. Ihr kritischer Ansatz ist damit eher nach innen gerichtet. Als Beispiel hierfür bestimmt das brasilianische Mietrecht zwar unmißverständlich, daß der Mieter eines Hauses dieses verlassen muß, wenn er den Mietzins nicht zu zahlen in der Lage ist, aber sog. alternative Richter haben sich auf die höherrangigen Verfassungsnormen und die in ihnen festgeschriebenen Grundrechte berufen, um - nicht selten gegen das spezielle Mietgesetz - arme und arbeitsunfähige Mieter im Besitz ihres Zuhauses zu erhalten, ohne daß diese die geschuldete Summe gezahlt hätten. Manche der Interpretationen dieser Richter (z.T. auch Staatsanwälte, Rechtsanwälte u.a.) sind allerdings derart extensiv, daß ihnen ihre Rechtswidrigkeit vorgeworfen wurde.

Alle diese drei Ansätze der Kritik am vorherrschenden dogmatischen Positivismus sind in den Augen einer vierten Tendenz nicht wirklich ausreichend; sie scheint weiter zu gehen und bringt das Konzept vom alternativen Recht mit einem politischen Kampf sowie der bevorzugten Ausrichtung auf die sozial benachteiligten Schichten in Verbindung, wenn sie zum Beispiel "die aktuelle Ebene des Klassenkampfes innerhalb der rechtlichen Instanzen" betont ${ }^{15}$. Die Diskussion über die Bewegung solle auf die Straße gehen und ihre volle

der höchste Gerichtshof vor kurzem gegenteilig; vgl. die Zeitschrift Veja (São Paulo) in der Ausgabe vom 22.5.1996 (Nr. 1445), S. 36. 
soziale Aufgabe erfüllen, denn "die Debatte verbleibt noch immer bloß akademisch und dringt nicht an die Bevölkerung oder die Arbeiterbewegung durch", welche die eigentlichen Adressaten dieser Formulierungen seien ${ }^{16}$. Andere Juristen allerdings sehen nicht gerade die Arbeiter als die hauptsächliche Zielgruppe alternativer Rechtspraxis, welche sich durch alle Sparten der Gesellschaft ziehen müsse; sie verstehen alternatives Recht in einem eher weiten Sinne als ideologische Bewegung, eine existentialistische Haltung jeglicher Form positiven Rechts gegenüber. In diesem Kontext kommt der Juristenausbildung eine entscheidende Rolle $\mathrm{zu}^{17}$.

Es ist jedenfalls bemerkenswert, daß es durchaus Formen alternativen Rechts gibt, die in hohem Maße von staatlichen Organen und Institutionen abhängen: Blickt man auf die systemische Umgebung, so sieht man seine Verfahren von öffentlichen Funktionären und Beamten gelenkt und erkennt den fruchtbaren Boden, den es in der Staatsbürokratie hat, schließlich versteht man sein Aufkommen auch vor dem Hintergrund einer ineffizienten Kompetenzverteilung im Staat. Eine erste Schlußfolgerung erscheint damit offensichtlich. Alle diese Formen schaffen eine Abhängigkeit zwischen den Teilsystemen und ermöglichen eine flüssigere Kommunikation zwischen Staat und Bevölkerung, indem sich der Staat über diese Strategien ständig und durch die ganze Gesellschaft hindurch präsent halten kann. Was das staatlich-dogmatische Recht betrifft, so mag die hier beschriebene Form gewohnheitlichen und außerdogmatischen Rechts in gleichem Maße seine Legitimation herausfordern wie - aber auch - dabei helfen, es an die Wirklichkeit anzupassen. (155). (FN 15), S. 143 - 154. 
execution on the other. The codes of conduct concerning the transfer of technology are not obligatory. Multilateral treatments determine technical cooperation and the transfer of financial ressources, added by procedural rules of compliance control, but these norms contain a large range of judgement, liability is controversial. The protection of intellectual property rights within the frame of international trade is suitable to stimulate further transfer of environmentally sound technology, it does not include specific obligations of transfer.

\section{Inconsistency Strategies in Peripheral Judicial Systems: A Form of Alternative Law}

\section{By João Maurício L. Adeodato}

This paper is based on a research on the Brazilian judicial system, concentrated in the Law Court of Recife, capital of the North-eastern federal State of Pernambuco. It is based on the epistemological assumption that societies which form the periphery of contemporary capitalism, imprecisely called underdeveloped, present a specific form of law. Our goal is to detect alternative forms of positive law, which, in a kind of friendly pluralism, cohabit with what we call dogmatic state law. Within these outbreaks of alternative law, we emphasise those proceedings which are, although clearly parallel to dogmatic state law or even contra legem, practised by State and government organs, themselves. This highly incoherent promiscuity surely presents problems, but it functions, as we have verified.

There has been a lot of talking about a law crisis, a State crisis and the like in contemporary peripheral societies. Perhaps a permanent state of crisis should not really be understood as crisis but rather as a form of organisation which might appear chaotic to some eyes and might, yet, operate very efficiently. For our purposes, Brazil could well exemplify this situation. Here, the social subsystems counteract mutually, interfere reciprocally with each other: The economic status of society and even of individuals, for example, plays a crucial role in the application of law and legitimisation of government; political casuistry and friendship relations can also modify the course of State decisions, a fact which is illustrated by the old saying: "to friends, everything, to adversaries, the law". Law becomes politicised or, more than that, allopoietic. It is our point that, among many difficulties to understand and describe this crisis, the pretence of extending to all countries the model of an eurocentered modern State plays a decisive part. As a basis for this approach, many jurists and other social scientists seem to start from a kind of ontological epistemology, i.e., the belief that it is possible to construct an universal theory, which could discover some kind of essence in law, politics, social relations, legitimisation, or in its forms of development or evolution. And this essence usually presents strong ties with what can be observed in the historical experience of the Welfare State. That is not always the case, though. 
It is interesting to report that there is a kind of alternative law which is largely dependent on state organs and institutions: looking at the surrounding systemic environment, its proceedings are conducted by public functionaries and officers, the fertile soil is the state bureaucracy, its needs arise from the inefficient distribution of State decisions; concerning dogmatic State law, this form of customary extra-dogmatic law may so much threaten its legitimisation as well as help adjusting it to reality. 